\title{
Gender and age effects in structural brain asymmetry as measured by MRI texture analysis
}

\author{
Vassili A. Kovalev, Frithjof Kruggel,* and D. Yves von Cramon \\ Max-Planck Institute of Cognitive Neuroscience, Stephanstrasse 1A, D-04103 Leipzig, Germany
}

Received 22 July 2002; revised 28 January 2003; accepted 12 February 2003

\begin{abstract}
Effects of gender and age on structural brain asymmetry were studied by 3D texture analysis in 380 adults. Asymmetry is detected by comparing the complex 3D gray-scale image patterns in the left and right cerebral hemispheres as revealed by anatomical T1-weighted MRI datasets. The Talairach and Tournoux parcellation system was applied to study the asymmetry on five levels: the whole cerebrum, nine coronal sections, 12 axial sections, boxes resulting from both coronal and axial subdivisions, and by a sliding spherical window of 9 mm diameter. The analysis revealed that the brain asymmetry increases in the anterior-posterior direction starting from the central region onward. Male brains were found to be more asymmetric than female. This gender-related effect is noticeable in all brain areas but is most significant in the superior temporal gyrus, Heschl's gyrus, the adjacent white matter regions in the temporal stem and the knee of the optic radiation, the thalamus, and the posterior cingulate. The brain asymmetry increases significantly with age in the inferior frontal gyrus, anterior insula, anterior cingulate, parahippocampal gyrus, retrosplenial cortex, coronal radiata, and knee region of the internal capsule. Asymmetry decreases with age in the optic radiation, precentral gyrus, and angular gyrus. The texture-based method reported here is based on extended multisort cooccurrence matrices that employ intensity, gradient, and anisotropy features in a uniform way. It is sensitive, simple to reproduce, robust, and unbiased in the sense that segmentation of brain compartments and spatial transformations are not necessary. Thus, it should be considered as another tool for digital morphometry in neuroscience.
\end{abstract}

(C) 2003 Elsevier Science (USA). All rights reserved.

Keywords: Structural brain asymmetry; MRI; Texture; Age; Gender

\section{Introduction}

The human brain is asymmetric in structure and function. Anatomical brain asymmetries were studied in vivo using various methods. Conventional approaches are based on volumetric measurements of gross interhemispheric asymmetry and specific anatomical structures (e.g., Yuecel et al., 2001; Kennedy et al., 1999; Amunts et al., 2000; Preis et al., 1999). Recently, several new technique based nonlinear image registration of whole brains have been proposed. The most prominent are voxel-based morphometry (Ashburner and Friston, 2000) and closely related methods (e.g., Good et al., 2001a,b; Davatzikos and Resnick, 1998; Gaser et al., 1999; Sowell et al., 1999; Davatzikos and Resnick, 2002).

\footnotetext{
* Corresponding author: Fax: +49-341-9940-221.

E-mail address: kruggel@cns.mpg.de (F. Kruggel).
}

Alternative, surface-based approaches characterize gyral and sulcal pattern variations across subjects in order to remove confounds of cortical surface variability (Thompson et al., 1997, 1998, 2001; Sowell et al., 2001, 2002; Narr et al., 2001a,b). Both approaches retain information about morphometric measures from individual subjects and facilitate the detection of fine structural differences in brain anatomy (e.g., local shape variation, local gray matter concentration, and asymmetry).

Quantification of structural brain asymmetry within precisely delineated regions requires segmentation of brain compartments and asymmetry assessment in terms of regional tissue composition (e.g., the gray-white matter (GWM) ratio). In $\mathrm{T}_{1}$-weighted MRI datasets, different brain tissue types are primarily attributed to certain overlapping intensity ranges in the image. An abundance of techniques for segmenting gray and white matter was proposed (e.g., 
Joliot and Mazoyer, 1993; Momenan et al., 1997; Rajapakse et al., 1997; Goldszal et al., 1998). However, providing a precise segmentation is difficult. As pointed out by Good et al. (2001b), GWM ratios in the range of 1 to 3 were reported in the literature. This broad range suggests that any segmentation method may introduce a bias on asymmetry measures. Explicit tissue classification is avoided by using 3D image descriptors that properly describe the complex spatial gray-scale structure of $\mathrm{T}_{1}$-weighted MRI brain datasets. Here, we employed image texture measures as captured by extended multisort cooccurrence matrices (Kovalev et al., 2001).

Because gray-scale patterns are examined, texture measures are based on certain subregions of the brain, rather than single voxels. We considered asymmetry measures on different spatial scales of the Talairach and Tournox (1988) coordinate system: the whole cerebrum, the nine coronal sections, the 12 axial sections, the box-shaped sections resulting from both coronal and axial subdivisions, and a sliding spherical window of $9 \mathrm{~mm}$ diameter. Because subdivisions were introduced in the native individual datasets, any nonlinear image registration and/or spatial scaling prior to the analysis is unnecessary, which would substantially affect the local image pattern. An increasing subregion size yields a higher sensitivity and a higher robustness of this texture measure, but at a lower spatial resolution. Because patterns are compared between subregions of both hemispheres, measures are symmetric with respect to the midsagittal plane.

On a database of $\mathrm{T}_{1}$-weighted MRI brain datasets acquired in 380 adults, texture measures were applied to study the interhemispheric asymmetry, their differences associated with gender, and changes with age. To the best of our knowledge, this is the first report assessing the structural brain asymmetry based on 3D texture analysis.

\section{Materials and methods}

\section{Subjects and groups}

The Max-Planck Institute of Cognitive Neuroscience maintains a database of subjects enrolled for functional MRI experiments. Before admission, a brief history and physical inspection is taken by a physician, and a high resolution $\mathrm{T}_{1}$-weighted MRI scan of the head is acquired. Subjects are included in this database if they comply with the informed consent for conducting general fMRI experiments, pass the examination, and do not exhibit pathological or abnormal features (e.g., unilateral ventricular enlargements, subarachnoidal cysts) in their MR tomograms. Scans used here were sampled from this database of normal volunteers, containing 516 subjects.

Brain asymmetry differences associated with gender were evaluated in a group of young healthy subjects (aged 18 to 32 years, denoted as GENDER). Pairs of male/female subjects were selected who differed in less than 1 year in age. This group consisted of 290 subjects ( 145 males, aged $24.1 \pm 2.6$ years; 145 females, aged $24.3 \pm 2.9$ years $)$. The age difference with gender was insignificant $(P=0.565)$ within this group.

Changes of brain asymmetry with age were studied in 152 healthy subjects (aged 18 to 70 years, denoted as AGE). Again, aged-matched pairs of male/female subjects were selected. To avoid an overrepresentation of young subjects, the number of pairs was limited to three per year and the maximum intrapair age difference was set to 2.5 years. This group included 76 males (aged $35.1 \pm 13.4$ years) and 76 females (mean age $36.1 \pm 14.1$ years). The age difference with gender was insignificant $(P=0.650)$ within this group.

As a result of the selection process, 62 subjects were included in both groups. Thus, the total number of subjects involved in our study was 380 .

\section{MRI scanning protocol and data preprocessing}

Magnetic resonance imaging was performed on a Bruker 3T Medspec 100 system, equipped with a bird cage quadrature coil, using a $\mathrm{T}_{1}$-weighted 3D MDEFT protocol (Lee et al., 1995): FOV $220 \times 220 \times 192 \mathrm{~mm}$, matrix $256 \times 256$, 128 sagittal slices, voxel size $0.9 \times 0.9 \mathrm{~mm}, 1.5 \mathrm{~mm}$ slice thickness, scanning time $15 \mathrm{~min}$. Scan data were interpolated to an isotropical voxel size of $1.0 \mathrm{~mm}^{3}$ using trilinear interpolation. The outer hulls of the brain were removed and brains were aligned with the Talairach coordinate system (Talairach and Tournoux, 1988) using an automated procedure (Kruggel and von Cramon, 1999).

\section{Brain regions}

The interhemispheric brain asymmetry was assessed using the Talairach coordinate system at different spatial scales:

- The whole cerebrum,

- The nine coronal sections $(x 1-x 9$, where $x 1$ corresponds to the most anterior slice),

- The 12 axial sections $(z 1-z 12$, where $z 1$ corresponds to the most superior slice),

- The 108 box-shaped sections from subdivision in the coronal and axial planes $(x 1 z 1-x 9 z 12)$,

- A sliding spherical window of $9 \mathrm{~mm}$ diameter $(n=$ 389 voxels).

Talairach sections and coordinates were determined individually for each brain without applying a spatial transformation to the image data.

\section{Cooccurrence image descriptors}

The structural brain asymmetry was assessed in $\mathrm{T}_{1^{-}}$ weighted MRI datasets by comparing 3D gray-scale patterns in corresponding subregions that are symmetric to the 
Table 1

Test images with simulated asymmetries and measured asymmetry values $\mathrm{A}$, and their interpretation

\begin{tabular}{|c|c|c|c|c|}
\hline No. & Test image & Simulated asymmetry & $\begin{array}{l}\text { Measured } \\
\text { asymmetry A }\end{array}$ & Comment \\
\hline 1 & & Reflected image patterns & 0 & $\begin{array}{l}\text { No asymmetry because image } \\
\text { descriptors are reflection invariant }\end{array}$ \\
\hline 2 & & Different global shape, same content & 0.009 & $\begin{array}{l}\text { Very little asymmetry because only } \\
\text { local voxel pairs are considered }\end{array}$ \\
\hline 3 & & Different size, similar content & 0.150 & Moderate asymmetry \\
\hline 4 & & Reduced gray-white volume ratio & 0.245 & $\begin{array}{l}\text { Severe asymmetry because the } \\
\text { local image pattern is different }\end{array}$ \\
\hline
\end{tabular}

midsagittal plane. We used extended multisort cooccurrence matrices (Kovalev et al., 2001) as detailed descriptors of the spatial image structure. These descriptors are computed from elementary features of voxel pairs within a subregion, such as intensity, intensity gradient magnitude (local intensity variation), the angle between gradient vectors (spatial coherence of intensity slopes), and the intervoxel distance. The cooccurrence matrix of an image subvolume is a multidimensional array. The matrix axes are associated with these features, and elements represent the number of voxel pairs with certain characteristics. All possible neighbors around each voxel with no repetition at a given distance are considered when computing matrices. Because all characteristics are relative, the cooccurrence matrix is invariant to translation, rotation, and reflection of image data.

Let us consider an arbitrary voxel pair $(i, k)$, defined on the discrete image lattice by voxel indices $i=\left(x_{i}, y_{i}, z_{i}\right), k$ $=\left(x_{k}, y_{k}, z_{k}\right)$ at Euclidean distance $d(i, k)$. Let us denote intensities of these voxels by $I(i)$ and $I(k)$, local gradient magnitudes by $G(i), G(k)$, and the angle between their intensity gradient vectors by $a(i, k)$. Then, the 6D cooccurrence matrix can be defined as

$$
W=\|w(I(i), I(k), G(i), G(k), a(i, k), d(i, k))\|,
$$

where $w$ corresponds to the number of voxel pairs $(i, k)$, with given intensity and gradient characteristics at the intervoxel distance $d(i, k)$, measured in integer bin units of 1 $\mathrm{mm}$ in size. Gradient magnitudes $G(i)$ and $G(k)$ were calculated from the orthogonal intensity gradient vector components $G_{x}, G_{y}$ and $G_{z}$ as

$$
\begin{aligned}
G(i) & =\sqrt{G_{x}^{2}(i)+G_{y}^{2}(i)+G_{z}^{2}(i)}, \\
G(k) & =\sqrt{G_{x}^{2}(k)+G_{y}^{2}(k)+G_{z}^{2}(k)} .
\end{aligned}
$$

Gradient vector components $G_{x}, G_{y}$, and $G_{z}$ were obtained by convolving the $3 \times 3 \times 3$ voxel neighborhoods with a Zucker-Hummel filter (1981). The angle between gradient vectors $a(i, k)$ of a voxel pair $(i, k)$ varies from 0 to 180 degrees and was calculated as

$$
a(i, k)=\cos ^{-1}(g(i) \circ g(k)),
$$

where $g(i) \circ g(k)$ denotes the dot vector product and $g(i)$, $g(k)$ are the normalized (unit) gradient vectors at voxel positions $i$ and $k$. The unit vectors $g(i), g(k)$ were calculated by dividing original vector components $G_{x}, G_{y}$, and $G_{z}$ by their magnitudes $G(i)$ and $G(k)$. Finally, elements of the cooccurrence matrix were normalized by the total sum of 


\section{gender}
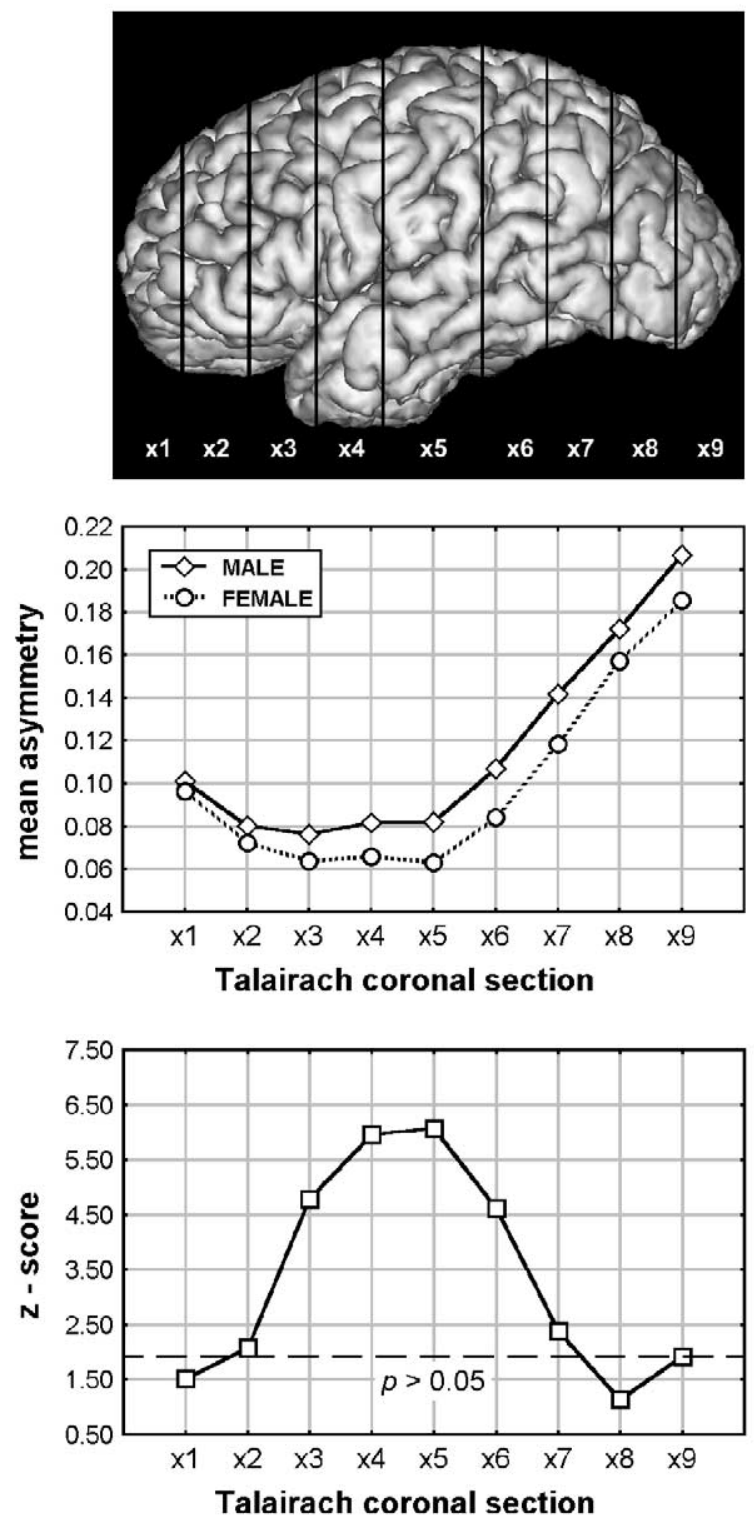

age
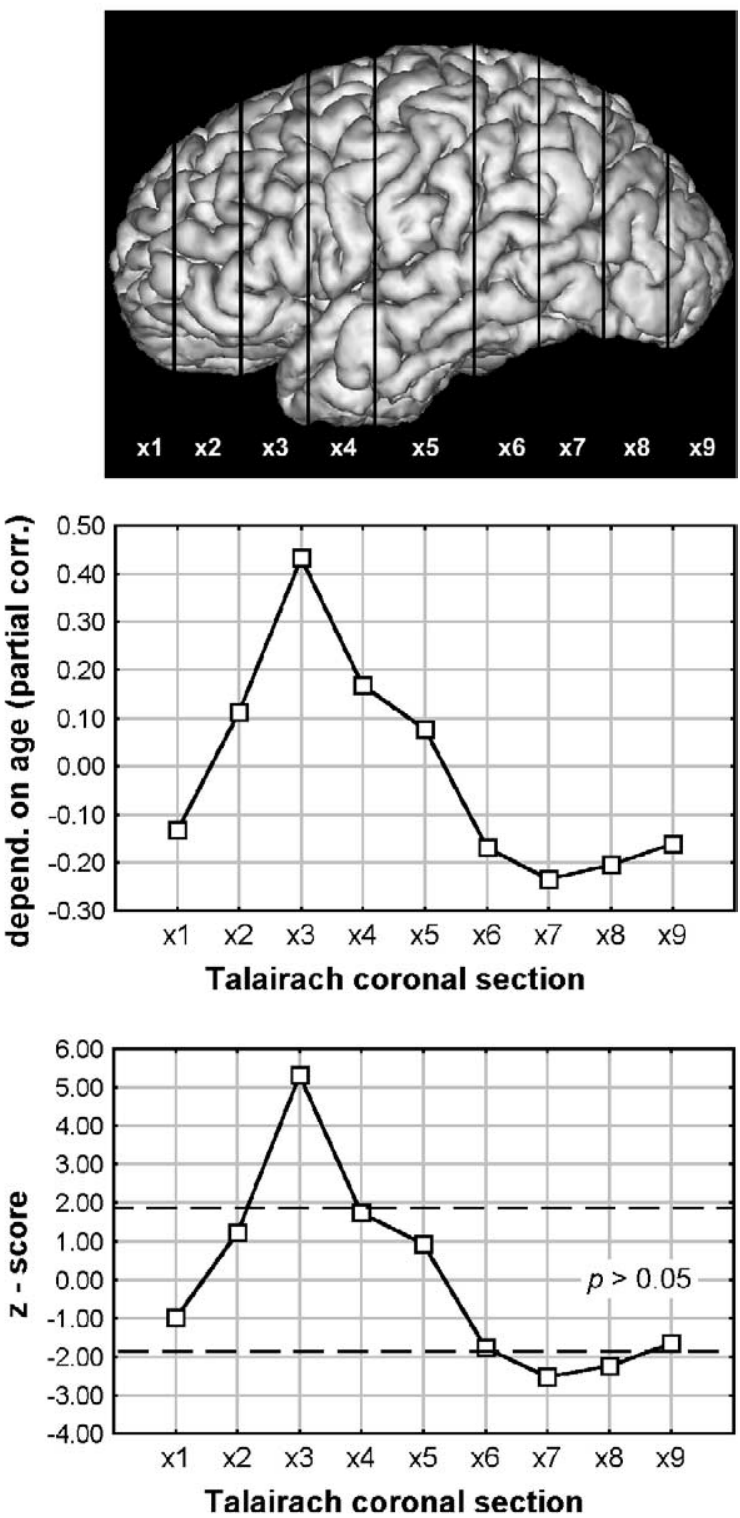

Fig. 1. Interhemispheric asymmetry differences in Talairach coronal sections associated with gender (left column, $n=290,145$ males, age range $18-32$ years) and asymmetry changes with age (right column, $n=152,76$ males, age range 18-70 years). Example brain subdivisions (top), interhemispheric asymmetry for male and female subjects (middle, left), partial correlation of the asymmetry with age (middle, right), and significance of asymmetry differences (below).

elements (which is proportional to the brain region volume) for each distance bin separately. Refer to Kovalev et al., 2001 for a more detailed description.

Intensity, gradient magnitude, and relative gradient orientation were chosen as cooccurrence matrix dimensions because they form a complementary set of elementary image features. If only voxel intensity were used, two voxel pairs with the same intensities $I(i)$ and $I(k)$ would be indistinguishable with respect to their location and therefore counted in the same matrix bin. Including the local gradient magnitude adds information about the homogeneity of the local neighborhood, i.e., whether the region is rather uniform in intensity (e.g., in the white matter) or has high intensity slopes (e.g., at the gray/white matter border). The gradient angle $a(i, k)$ mostly captures gyral and sulcal shape variability.

\section{Asymmetry measure}

Once the cooccurrence matrices of two regions in the left and right brain hemispheres were calculated, their distance in the high-dimensional feature space is defined as the asymmetry measure. We used the normalized sum of absolute element-by-element differences (commonly called L1norm). For two cooccurrence matrices $W^{L}$ and $W^{R}$ with $M$ elements, the asymmetry measure $A$ is calculated as 
gender
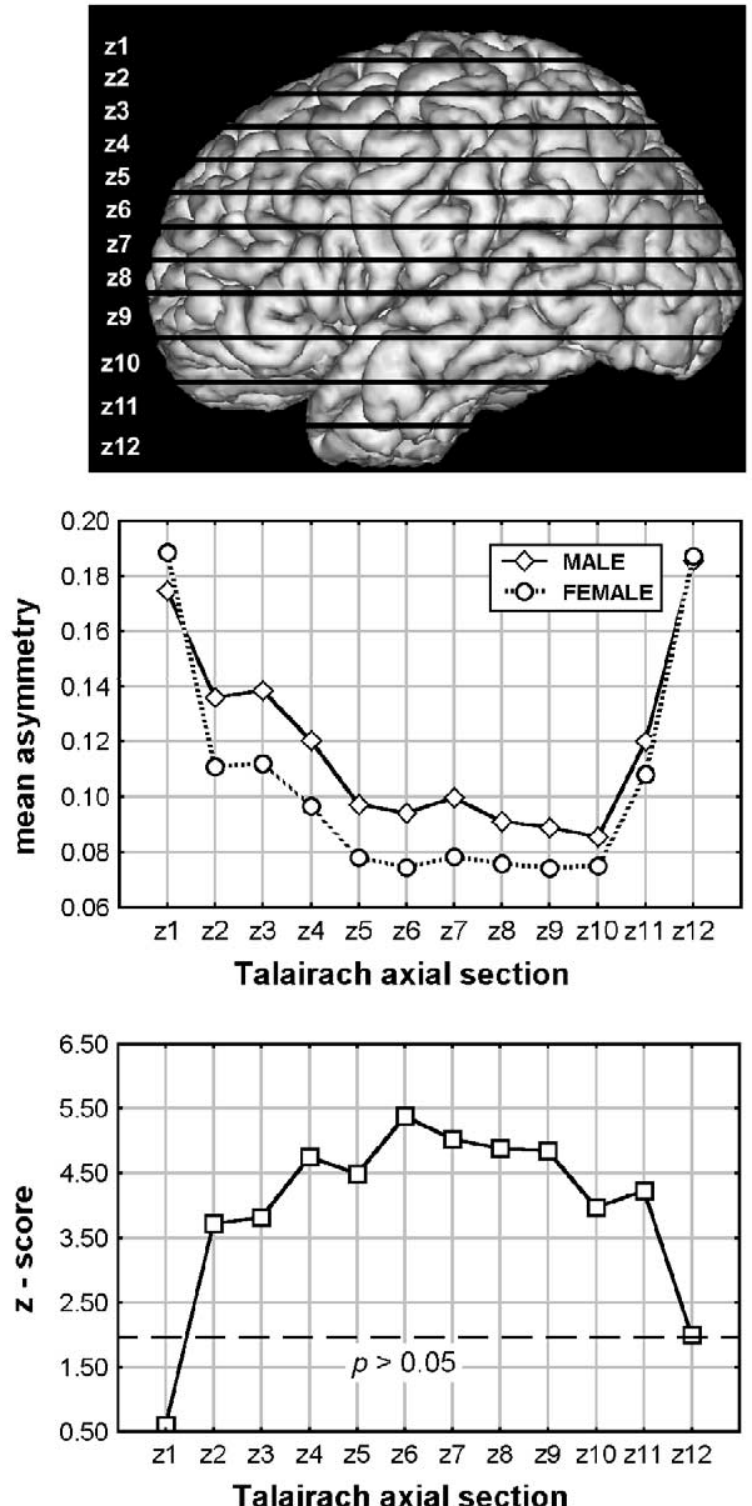

age
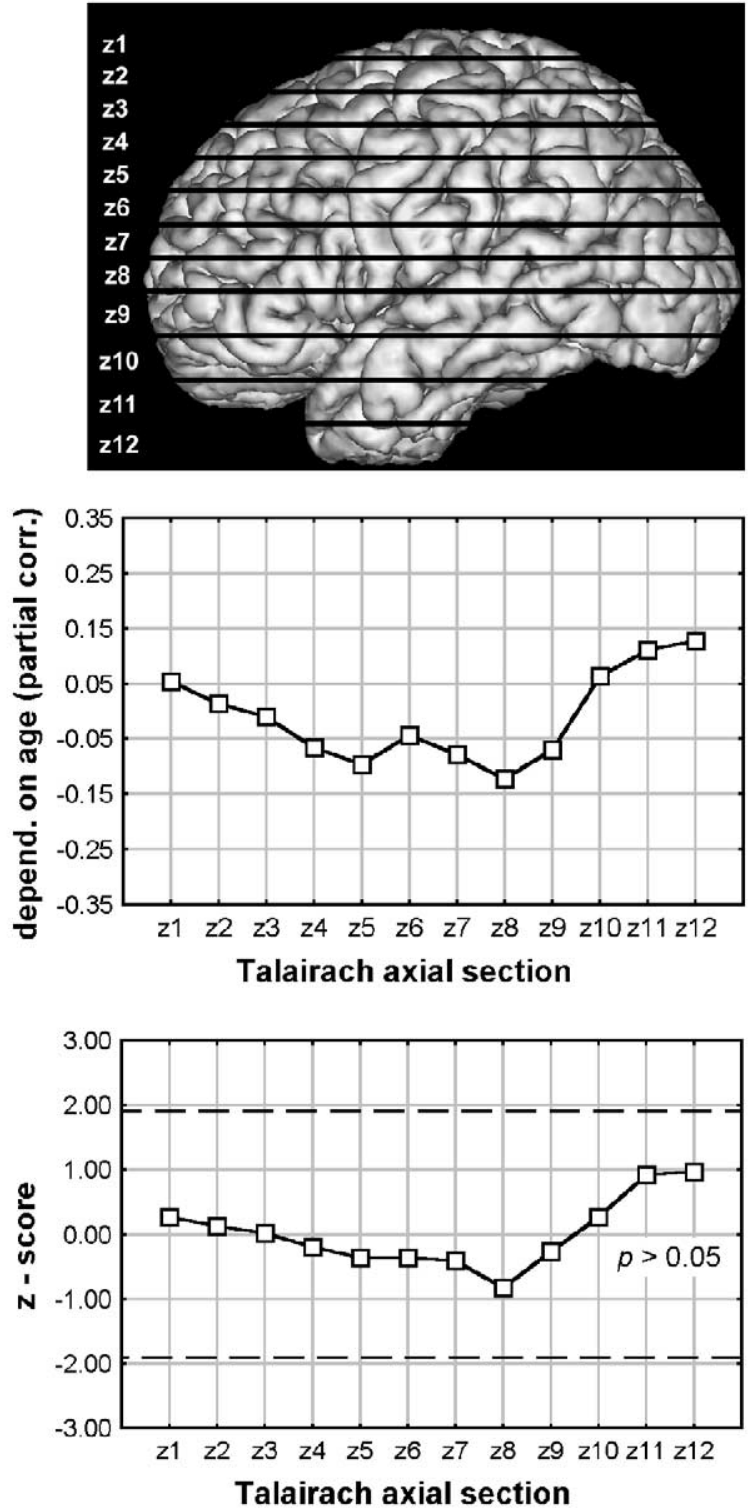

Fig. 2. Interhemispheric asymmetry differences in Talairach axial sections associated with gender (left column, $n=290,145$ males, age range 18-32 years) and asymmetry changes with age (right column, $n=152,76$ males, age range 18 -70 years). Example brain subdivisions (top), interhemispheric asymmetry for male and female subjects (middle, left), partial correlation of asymmetry with age (middle, right), and significance of asymmetry differences (below).

$$
A\left(W^{L}, W^{R}\right)=A\left(W^{R}, W^{L}\right)
$$

$$
=L 1\left(W^{L}, W^{R}\right)=\frac{\sum_{j=1}^{M}\left|w_{j}^{L}-w_{j}^{R}\right|}{\sum_{j=1}^{M} w_{j}^{L}+\sum_{i=1}^{M} w_{j}^{R}} .
$$

Remember that this measure expresses the dissimilarity of two image patterns and is thus unsigned. It ranges from 0 (identical patterns) to 1 (very different patterns).

\section{Control parameters}

Bin sizes of cooccurrence matrices are important control parameters (Clausi, 2002). Optimal binning parameters for intensity, gradient magnitude, and gradient angle were selected experimentally in a prestudy. We varied the bin count from 2 to 128 bins for intensity and gradient magnitude and from 2 to 12 for the relative gradient angle. For each set of control parameters, cooccurrence descriptors of brain hemispheres of 290 subjects of group GENDER were calculated and the significance of gender-related asymmetry differences was evaluated. A significance decrease was found for 


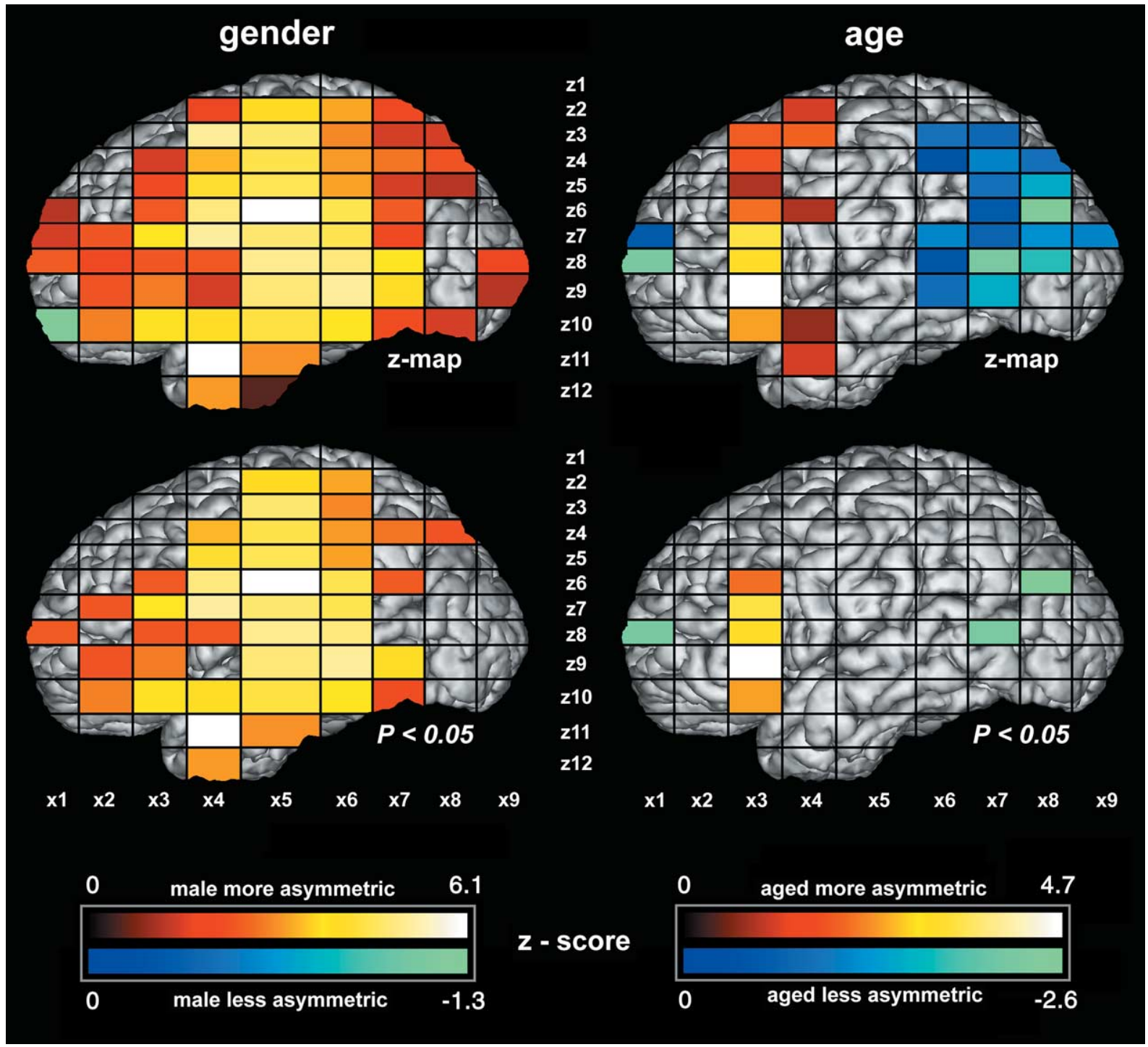

Fig. 3. Significance of interhemispheric asymmetry differences in Talairach coronoaxial sections associated with gender (left column, $n=290,145$ males, age range 18 -32 years) and significance of asymmetry changes with age (right column, $n=152,76$ males, age range $18-70$ years), $z$ score maps (top: uncorrected, below: corrected for $n=9$ comparisons).

Table 2

Most prominent regions of gender-related asymmetries

\begin{tabular}{lll}
\hline Anatomical region & Talairach coordinates & $z$ score \\
\hline Lateral orbitofrontal gyrus & $-21,18,-13$ & 5.0 \\
Temporal stem & $-38,-51,-5$ & 5.0 \\
Thalamus & $-12,-35,6$ & 4.9 \\
Superior temporal gyrus & $-36,-21,6$ & 5.0 \\
Heschl's gyrus & $-37,-31,12$ & 5.0 \\
Optic radiation, knee & $-25,-36,12$ & 5.0 \\
Dorsomedial thalamus & $-6,-23,14$ & 5.0 \\
Temporal operculum & $-40,-9,18$ & 4.7 \\
Posterior cingulate & $-11,-49,27$ & 5.0 \\
Precentral gyrus & $-42,-19,38$ & 4.9 \\
Posterior frontal white matter & $-16,-21,-53$ & 5.0 \\
\hline
\end{tabular}

Table 3

Most prominent regions of asymmetry changes with age

\begin{tabular}{llc}
\hline Anatomical region & Talairach coordinates & $z$ score \\
\hline Parahippocampal gyrus & $-22,-50,-15$ & 5.0 \\
Inferior frontal gyrus & $-40,32,-7$ & 3.6 \\
Anterior cingulate & $-17,33,6$ & 5.0 \\
Anterior insula & $-30,16,6$ & 5.0 \\
Internal capsule, knee & $-13,-3,9$ & 5.0 \\
Retrosplenial cortex & $-7,-71,12$ & 3.2 \\
Corona radiata & $-20,-5,23$ & 5.0 \\
Optic radiation & $-35,-69,-7$ & -2.9 \\
Precentral gyrus & $-6,-4,21$ & -3.4 \\
Angular gyrus & $-57,-56,23$ & -3.3 \\
\hline
\end{tabular}




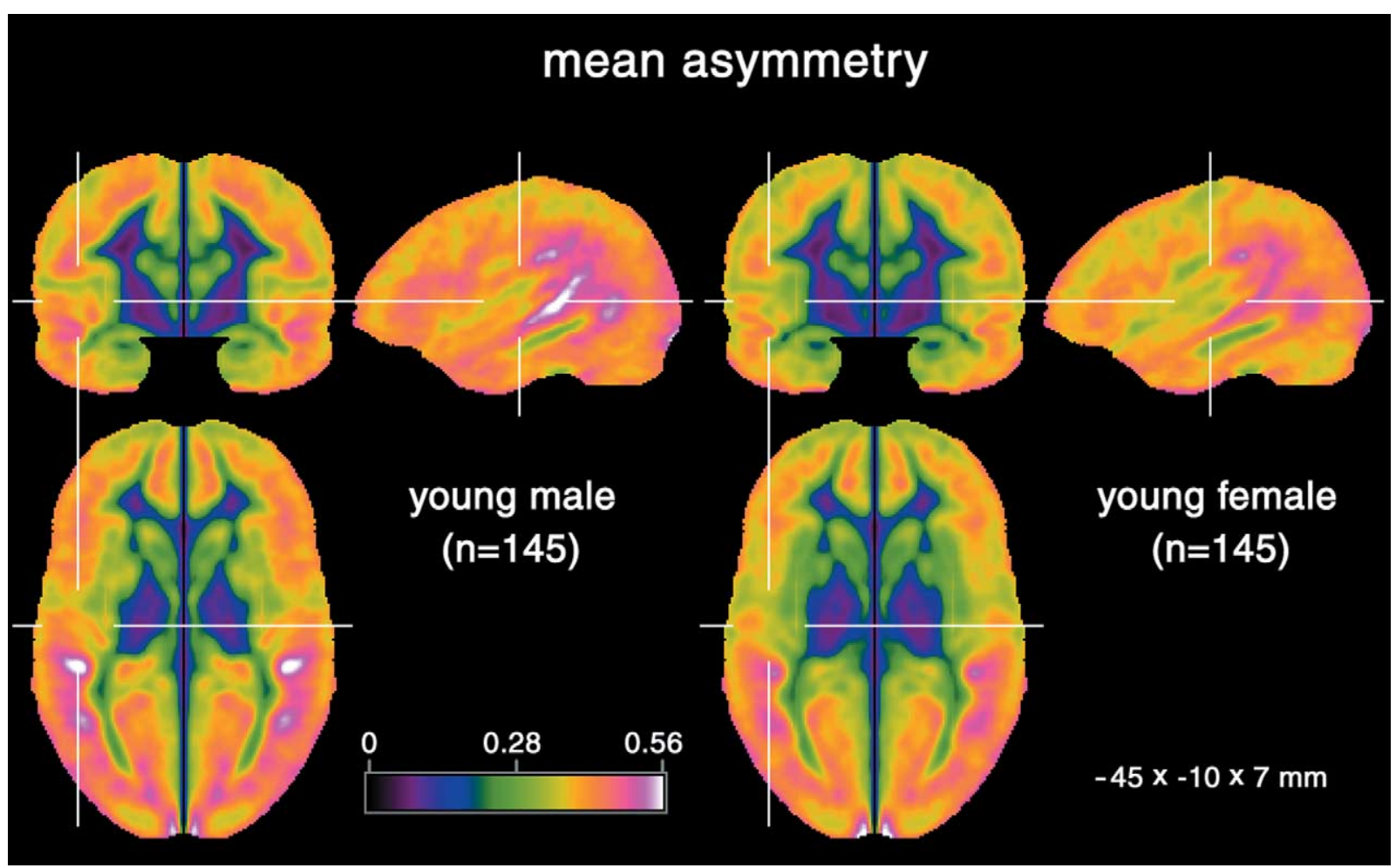

Fig. 4. Orthogonal sections of mean asymmetry maps for young males (left panel, $n=145,18-32$ years) and females (right panel, $n=145,18-32$ years). Note the prominent asymmetry in males appearant in the vicinity of Heschl's gyrus, the superior temporal gyrus, postcentral gyrus, and the optic radiation. In females (as in males), the only prominent asymmetry is found at the occipital pole, and much less at the postcentral gyrus.

more than eight intensity bins. Thus, the number of intensity (and, consequently, gradient magnitude bins) was set to 8 . Collecting gradient angles in six bins was found to be a good compromise between resolution and robustness. The maximal intervoxel distance $d(i, k)$ was set to one image raster unit, corresponding to an average neighborhood diameter of $4.26 \mathrm{~mm}$ involved in the calculation of the cooccurrence descriptors. Note that interscan differences in the intensity scale are not considered as critical, because asymmetry measures were derived within a scan, normalized, and then compared across the group.

\section{Illustrative examples}

In this section, we provide an illustration of key aspects of the method with the help of synthetic images, simulating different types of interhemispheric asymmetries (Table 1). The original 2D images of $280 \times 128$ pixels in size were converted to pseudovolumetric datasets with three identical slices. Then, the cooccurrence descriptors for the left and right halves and the asymmetry values were calculated as described above.

The first example illustrates the reflection invariance of image descriptors. The second one demonstrates the low sensitivity of measures to global shape differences. The example provided in the third row of Table 1 simulates the influence of gross size differences; the right half was scaled down to $75 \%$. Ideally, the asymmetry measure should be close to zero here due to the normalization. A moderate asymmetry is observed with this size reduction for two reasons: (1) the voxel sampling rate is kept constant for both halves and not reduced for the right half accordingly, (2) due to scaling, some intensity information was inevitably lost. Finally, the fourth row of Table 1 illustrates the high sensitivity of the asymmetry measure to differences in the GWM ratio. Changes of GWM ratio lead to a substantial redistribution of intensity bin counts of cooccurrence descriptors, and consequently, to a high asymmetry value.

\section{Implementation}

The calculation of cooccurrence descriptors and asymmetry values for MRI- $\mathrm{T}_{1}$ brain datasets were implemented in the $\mathrm{C}$ programming language on a $\mathrm{PC}$ workstation with two Intel P-III $750 \mathrm{MHz}$ processors and 1GB RAM running under the Linux operating system.

\section{Statistical analysis}

The significance of asymmetry differences was assessed statistically by fitting multivariate linear models to the data. Gender, age, and subregion volume were included as factors explaining brain asymmetry. Significance scores for the gender factor were reported for the GENDER group and scores of the age factor are discussed when evaluating the 
AGE group. The definition of the subregion volume corresponds to the spatial scale of the analysis. The entire cerebral volume was used at the first level; volumes of Talairach coronal and axial sections and coronoaxial boxes were entered to the multivariate model as third factor when assessing structural asymmetry in these sections separately. Finally, on the regional level of the analysis the volume was measured as a portion of brain within the spherical voxel neighborhood.

All significance values were corrected for multiple comparisons using Holm's method (1979) and converted into $z$ scores to ease comparisons. Significance levels for twotailed $t$ statistics were set to $P<0.05$ (or $|z|>1.96$ ). In all the analyses, except for the whole cerebrum, significance values were corrected for multiple comparisons. The statistical software package R (Becker et al., 1988; Chambers and Hastie, 1992) was used for all evaluations.

\section{Results}

\section{Asymmetry of brain hemispheres}

First, the influence of age and gender on brain asymmetry was studied on the most coarse level, using whole cerebral hemispheres as subregions. The significance of asymmetry differences was statistically assessed using multivariate linear models with gender, age, and cerebral volume as factors. The asymmetry differences associated with gender were evaluated using group GENDER of 290 young healthy subjects. Male brains are significantly more asymmetric than female brains (male: $0.081 \pm$ 0.037; female: $0.061 \pm 0.028, z=5.52, P<3.5 \times$ $10^{-8}$ ). When excluding brain volume as a factor, the significance score is lower $\left(z=4.61, P<4.0 \times 10^{-6}\right)$. Male brains are significantly greater than female brains (male: $1282 \pm 93 \mathrm{ml}$; female: $1161 \pm 87 \mathrm{ml}, z=10.35$, $\left.P<10^{-8}\right)$. Changes of interhemispheric asymmetry with age were determined using group AGE of 152 gendermatched subjects in the range of 18 to 70 years. Changes with age were not significant $(z=-0.58, P=0.558)$, even when male and female subjects were entered separately (male: $P=0.887$, female: $P=0.449$ ). At a gross level of cerebral hemispheres, male brains are significantly more asymmetric than female brains. This finding is independent of the significant difference in brain volume. In contrast, no global interhemispheric asymmetry changes were found with age.

\section{Asymmetry in Talairach sections}

\section{Coronal sections}

Gender- and age-related asymmetry differences were evaluated in nine Talairach coronal sections. Note that the subregion volume varied from $35 \mathrm{ml}$ in section $\mathrm{x} 1$ to $314 \mathrm{ml}$ in section $\mathrm{x} 5$. Asymmetry differences associated with gender are shown in the left column of Fig. 1. Again, the mean asymmetry values are higher in males than in females. These differences are significant in all sections except $\mathrm{x} 1(z=1.52, P=0.130)$, $\mathrm{x} 8(z=1.15, P=0.251)$, and $\mathrm{x} 9(z=1.93, P=0.054)$. The highest significance scores for gender effect are found in sections $\mathrm{x} 4\left(z=5.96, P<1.0 \times 10^{-6}\right)$ and $\mathrm{x} 5(z=6.08, P<$ $\left.1.0 \times 10^{-6}\right)$. Changes with age in coronal sections are shown in the right column of Fig. 1. The asymmetry increases significantly with age in section $x 3\left(z=5.33, P<1.0 .3 \times 10^{-6}\right)$, while it declines in posterior sections (x7: $z=-2.53, P=$ 0.011 ; 8 : $z=-2.25, P=0.024$ ).

\section{Axial sections}

Gender- and age-related asymmetry differences were evaluated in 12 Talairach coronal sections. The subregion volume varied from $12 \mathrm{ml}$ in section z12 to $187 \mathrm{ml}$ in section z9. The interhemispheric asymmetry was significantly greater in males in all axial sections except z1 (see Fig. 2, left). Maximal scores were achieved in sections z6 ( $z$ $\left.=5.38, P<1.0 \times 10^{-6}\right)$ and $\mathrm{z} 7(z=5.03, P<1.0 \times$ $\left.10^{-6}\right)$. No age-related effect was found in all 12 axial sections (see Fig. 2, right). The relatively even distribution of asymmetry differences with gender confirms the global brain asymmetry in males.

\section{Coronoaxial sections}

We considered interhemispheric brain asymmetry in 108 box-shaped regions from subdividing in both coronal and axial Talairach planes. Due to natural intersubject variability of brain shape, some $\mathrm{x}-\mathrm{z}$ sections were empty for some subjects. These sections were marked as "not available" in the statistics, thereby reducing statistical power. Genderrelated effects are shown in Fig. 3, left, as color-coded $z$ score maps superimposed on the $\mathrm{x}-\mathrm{z}$ Talairach grid. The upper map includes significance scores for all sections while the map below shows sections with significant asymmetry differences only $(P<0.05)$. Due to a greater brain asymmetry in males, $z$ scores are positive in all sections except x1z10 $(z=-1.34, P=0.179)$. The highest positive scores were found at the inferior part of the central sulcus (section x5z6, male: 0.124 , female $0.099, z=6.08, P<1.0 \times 10^{-6}$ ) and at the anterior tip of the temporal lobe (section $\mathrm{x} 4 \mathrm{z} 11$, male: 0.194 , female $0.167, z=5.89, P<1.0 \times 10^{-6}$ ). Age-related changes are shown in Fig. 3, right. Here, positive significance scores correspond to sections where the asymmetry increases with age. The highest positive scores were found at the inferior part of the inferior frontal gyrus (section $\times 3 z 9, z=4.74, p<2.2 \times 10^{-6}$, and $\times 3 z 8, z=$ $3.17, P=0.001)$ and the highest negative scores at the parietooccipital transition (section $\mathrm{x} 8 \mathrm{z6}, z=-2.56, P=$ 0.010 , and $\mathrm{x} 7 \mathrm{z} 8, z=-2.21, P=0.027)$.

\section{Regional asymmetry}

Pairs of spherical regions (9 $\mathrm{mm}$ diameter, 389 voxels) were selected symmetrically with respect to the midsagittal 
plane in the Talairach grid. Coordinates of the central voxels were mapped back into the individual datasets. Regional brain asymmetry values were computed, assigned to their central voxel, and assessed as described above. Results are presented in Fig. 5 as 3D maps of the mean asymmetry for male and female brains. Fig. 5 compiles $z$ score maps for gender-related differences (left column) and age-related changes (right column). The top panel displays characteristic positions of highly significant areas (Rorden and Brett, 2000). The bottom panel depicts significance scores superimposed onto four axial slices with Talairach coordinates provided for regions highlighted by crossed lines. The Talairach coordinates of specific brain regions with several highly significant gender-related difference are provided in Table 2, and for age-related effects in Table 3. Results presented in Figs. 4 and 5 are in agreement with those obtained for coronoaxial Talairach blocks but provide higher spatial resolution.

\section{Discussion}

This study assessed the asymmetry of the human brain as revealed by anatomical MRI. Two large subject groups were sampled, one to reveal gender-related differences, the other to study changes with age. Brain subregions of different size were selected in a single subject, and the image pattern within this region was characterized by a set of texture feature measures. The normalized L1-difference of these measures for corresponding subregions in both hemispheres was used as the asymmetry measure for this subregion. Thus, image analysis takes place at the original resolution of the acquired data, without any prior segmentation or nonlinear resampling. Subregions were defined on the grid and coordinate system defined by Talairach and Tournoux (1988), and asymmetry measures from individual datasets mapped into this common space for statistical analysis. Because our method effectively compares the image pattern in corresponding brain regions, the obtained asymmetry measure is unsigned, i.e., mapped values are symmetric w.r.t. the midsagittal plane (see Fig. 4).

In our study, the brain asymmetry in anterior-posterior direction exhibited a J-shaped curve (see Fig. 1, middle left) with an increase starting at Talairach coronal section $\mathrm{x} 5$ (i.e., behind the central sulcus). This result is well in agreement with several findings: The trajectory of the posterior ramus of the Sylvian fissure is steeper in the right hemisphere (Loftus et al., 1993; Sowell et al., 2001; Narr et al., 2001b; Thompson et al., 2001). Language-related cortices in the perisylvian region (e.g., the planum temporale, superior and inferior temporal cortex) are larger in the left hemiphere (Narr et al., 2001b; Thompson et al., 2001). The left occipital lobe and the right frontal lobe are larger than the opposite lobes in the majority of individuals (Chiu and Damasio, 1980; Kennedy et al., 1999). The frontal asymmetry was indicated here as a slightly higher asymmetry for section $x 1$ (see Fig. 1). The region-specific maps (see Fig. 4) confirm these results; high asymmetry values were found in the perisylvian region, along the central sulcus, the optical radiation, and at the occipital pole.

Although the pattern of asymmetries is similar in males and females (Fig. 4), values are higher and more focused in specific regions in males (see also Table 2). It is well known that male brains are more asymmetric than female brains. Yuecel et al. (2001) reported a consistently larger right hemisphere in males, while females had approximately equally sized hemispheres. The slope of the superior temporal sulcus in the right hemisphere is significantly steeper in males (Narr et al., 2001b). Good et al. (2001b) reported a significant leftward asymmetry of the gray matter volume in males at the medial end of Heschl's gyrus, and at the junction with the planum temporale, and a significant gender-related difference in adjacent white matter regions. The left motor cortex is significantly thicker in right-handed males but not in females (Amunts et al., 2000). Evaluating the significance of gender-related differences in our data confirmed the higher asymmetry for the following regions in males (see Fig. 5 and Table 2): Heschl's gyrus and the adjacent superior temporal gyrus, the temporal stem, the knee of the optic radition, and the precentral gyrus. As a correlate of the asymmetries, the thalamus and the posterior cingulate were also found as more asymmetric in males. Further regions of gender-related differences were found at the lateral orbitofrontal gyrus and the temporal operculum. The marked asymmetry in the posterior frontal white matter is also seen in the difference of the mean asymmetry values for axial slices z2 and z3 (see Fig. 2). All reported regions are in close correspondence with results reported by Good et al. (2001b), although the spatial resolution is higher here, due to a methodological advantage.

Changes of asymmetry with age were much less prominent. While no global interhemispheric asymmetry changes were found with age, the more region-specific analyses revealed significant changes in the inferior frontal gyrus, the anterior insula, and the anterior cingulate (see Fig. 5, right and Table 3). These three regions together marked only coronal section x3 (see Fig. 1) resp. boxes x3z6-x3z10 (Fig. 3 , right) as asymmetric. This confirms results by Sowell et al. (2002), who found the most prominent area of asymmetry in adult subjects in the inferior prefrontal cortex. Memory-related cortices (e.g., the parahippocampal gyrus and the retrosplenical cortex, Table 3 ) and gross white matter bundles (e.g., the corona radiata and the knee region of the internal capsule) become more asymmetric with age. Some of the marked asymmetric regions discussed above become less asymmetric during life: the optic radiation, the precentral gyrus, and the angular gyrus (see Fig. 5, right and Table $3)$. Results are compatible with reports by Good et al. (2001a), using voxel-based morphometry. Similar changes of white matter structures with age were also reported by Davatzikos and Resnick (2002): an age-related asymmetry increase in the white matter of the left frontal lobe, resp. a decrease in the parietooccipital white matter compartment.

Of course, all left-right asymmetries discussed here must 


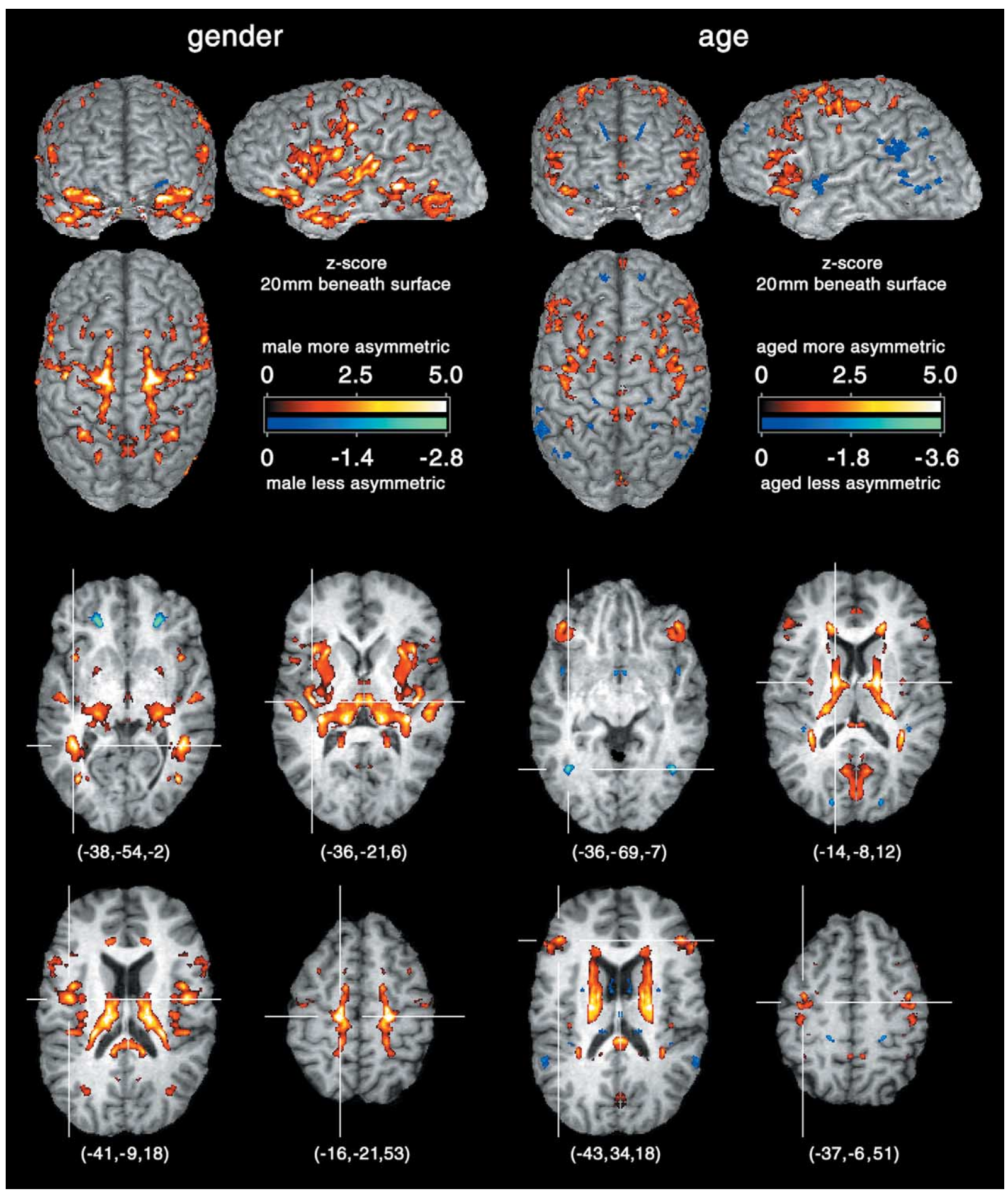

Fig. 5. Significance of regional asymmetry differences associated with gender (left column, $n=290,145$ males, age range 18-32 years) and significance of asymmetry changes with age (right column, $n=152,76$ males, age range 18 -70 years). $z$ Score values 20 mm beneath the brain surface (top) and example axial slices with superimposed $z$ score values (below). Talairach coordinates are provided for sample regions.

be weighted in terms of the individual structural variability of the human brain. There is a well-known, considerable shape variability of both frontal lobes and the temporooccipital gyri (Thompson et al., 2001). This variability increases asymmetry values, but spoils significance values. An example: the less-variable sections in the vicinity of the central sulcus receive low asymmetry values, but are of a rather high significance. Vice versa, the relatively variable frontal and temporo-parieto-occipital areas are asymmetric, but this effect is of less significance. Thus, we consider that our results represent more the individual asymmetry than the structural variability in a group. 
In summary, our findings are in line with other more region-specific (e.g., focusing on a few brain structures) and global approaches (e.g., focusing on surface properties). The texture-based method reported in this work is computationally inexpensive, simple to reproduce, and robust. The high significance scores and the ability to detect age-related asymmetry changes even in a group of young subjects is consistent with the conclusion of Bernasconi et al. (2001) regarding the high sensitivity of texture-based techniques. The method is unbiased in the sense that segmentation of brain compartments and spatial transformations are not necessary. Thus, texture-based methods may be considered as additional tools for digital morphometry in neuroscience.

\section{References}

Amunts, K., Jaenke, L., Mohlberg, H., Steinmetz, H., Zilles, K., 2000. Interhemispheric asymmetry of the human motor cortex related to handedness and gender. Neuropsychologia 38, 304-312.

Ashburner, J., Friston, K.J., 2000. Voxel-based morphometry-the methods. Neuroimage 11, 805-821.

Becker, R.A., Chambers, J.M., Wilks, A.R., 1988. The New S language Chapman \& Hall, New York.

Bernasconi, A., Antel, S.B., Collins, D.L., Bernasconi, N., Olivier, A., Dubeau, F., Pike, G.B., Andermann, F., Arnold, D.L., 2001. Texture analysis and morphological processing of magnetic resonance imaging assist detection of focal cortical dysplasia in extra-temporal partial epilepsy. Ann. Neurol. 49, 770-775.

Chambers, J.M., Hastie, T.J. (Eds.), 1992. Statistical Models in S. Chapman \& Hall, New York.

Chiu, H.C., Damasio, A.R., 1980. Human cerebral asymmetries evaluated by computed tomography. J Neurol. Neurosurg. Psychol. 43, 873-878.

Clausi, D.A., 2002. An analysis of co-occurrence texture statistics as a function of grey level quantization. Can. J. Remote Sensing 28, 45-62.

Davatzikos, C., Resnick, S.M., 1998. Sex differences in anatomic measures of interhemispheric connectivity: correlations with cognition in women but not men. Cereb. Cortex 8, 635-640.

Davatzikos, C., Resnick, S.M., 2002. Degenerative age changes in white matter connectivity visualized in vivo using magnetic resonance imaging. Cereb. Cortex 12, 767-771.

Gaser, C., Volz, H.P., Kiebel, S., Riehemann, S., Sauer, H., 1999. Detecting structural changes in whole brain bases on nonlinear deformationsapplication to schizophrenia research. Neuroimage 10, 107-113.

Goldszal, A.F., Davatzikos, C., Pham, D.L., Yan, M.X.H., Bryan, R.N., Resnick, S.M., 1998. An image processing protocol for qualitative and quantitative volumetric analysis of brain images. J. Comput. Assist. Tomogr. 22, 827-837.

Good, C.D., Johnsrude, I.S., Ashburner, J., Henson, R.N.A., Friston, K.J., Frackowiak, R.S.J., 2001a. A voxel-based morphometric study of ageing in 465 normal adult human brains. Neuroimage 14, 21-36.

Good, C.D., Johnsrude, I.S., Ashburner, J., Henson, R.N.A., Friston, K.J., Frackowiak, R.S.J., 2001b. Cerebral asymmetry and the effects of sex and handedness on brain structure A voxel-based morphometric analysis of 465 normal adult human brains. Neuroimage 14, 685-700.

Holm, S., 1979. A simple sequentially rejective multiple test procedure. Scand. J. Statistics 6, 65-70.

Joliot, M., Mazoyer, B.M., 1993. Three-dimensional segmentation and interpolation of magnetic resonance brain images. IEEE T Med. Imag. $12,269-277$

Kennedy, D.N., O'Craven, K.M., Ticho, B.S., Goldstein, A.M., Makris, N., Henson, J.W., 1999. Structural and functional brain asymmetries in human situs inversus totalis. Neurology 53, 1260-1265.
Kovalev, V.A., Kruggel, F., Gertz, H.-J., von Cramon, D.Y., 2001. Threedimensional texture analysis of MRI brain datasets. IEEE T Med. Imag. 20, 424-433.

Kruggel, F., von Cramon, D.Y., 1999. Alignment of magnetic-resonance brain datasets with the stereotactical coordinate system. Med. Image Anal. 3, 175-185.

Lee, J.H., Garwood, M., Menon, R., Adriany, G., Andersen, P., Truwit, C.L., Ugurbil, K., 1995. High contrast and fast three-dimensional magnetic resonance imaging at high field. Magn. Reson. Med. 34, $308-312$.

Loftus, W.C., Tramo, M.J., Thomas, C.E., Green, R.L., Nordgren, R.A., Gazzaniga, M.S., 1993. Three-dimensional quantitative analysis of hemispheric asymmetry in the human superior temporal region. Cereb. Cortex 3, 348-355.

Momenan, R., Hommer, D., Rawlings, R., Ruttimann, U., Kerich, M., Rio, D., 1997. Intensity-adaptive segmentation of single-echo T1-weighted magnetic resonance images. Hum. Brain Mapp. 5, 194-205.

Narr, K.L., Thompson, P.M., Sharma, T., Moussai, J., Blanton, R., Anvar, B., Edris, A., Krupp, R., Rayman, J., Khaledy, M., Toga, A.W., 2001a. Three-dimensional mapping of temporo-limbic regions and the lateral ventricles in schizophrenia: gender effects. Biol. Psychiatry 50, 84-97.

Narr, K.L., Thompson, P.M., Sharma, T., Moussai, J., Zoumalan, C., Rayman, J., Toga, A.W., 2001b. Three-dimensional mapping of gyral shape and cortical surface asymmetries in schizophrenia: gender effects. Am. J. Psychiatry 158, 244-255.

Preis, S., Jancke, L., Schmitz-Hillebrecht, J., Steinmetz, H., 1999. Child age and planum temporale asymmetry. Brain Cogn. 40, 441-452.

Rajapakse, J.C., Giedd, J.N., Rapoport, J.L., 1997. Statistical approach to segmentation of single-channel cerebral MR images. IEEE T Med. Imaging 16, 176-186.

Rorden, C., Brett, M., 2000. Stereotaxic display of brain lesions. Behav. Neurol. 12, 191-200.

Shaffer, J.P., 1995. Multiple hypothesis testing. Ann. Rev. Psychol. 46, 561-576.

Sowell, E.R., Thompson, P.M., Holmes, C.J., Batth, R., Jernigan, T.L., Toga, A.W., 1999. Localizing age-related changes in brain structure between childhood and adolescence using statistical parametric mapping. Neuroimage 9, 587-597.

Sowell, E.R., Thompson, P.M., Tessner, K.D., Toga, A.W., 2001. Mapping continued brain growth and gray matter density reduction in dorsal frontal cortex: inverse relationships during postadolescent brain maturation. J. Neurosci. 21, 8819-8829.

Sowell, E.R., Thompson, P.M., Rex, D., Kornsand, D., Tessner, K.D., Jernigan, T.L., Toga, A.W., 2002. Mapping sulcal pattern asymmetry and local cortical gray matter distribution in vivo: maturation in perisylvian cortices. Cereb. Cortex 12, 17-26.

Talairach, J., Tournoux, P., 1988. Co-planar Stereotactic Atlas of the Human Brain. Thieme, Stuttgart.

Thompson, P.M., MacDonald, D., Mega, M.S., Holmes, C.J., Evans, A.C., Toga, A.W., 1997. Detection and mapping of abnormal brain structure with a probabilistic atlas of cortical surfaces. J. Comp. Assist. Tomogr. $21,567-581$.

Thompson, P.M., Moussai, J., Zohoori, S., Goldkorn, A., Khan, A.A., Mega, M.S., Small, G.W., Gummings, J.L., Toga, A.W., 1998. Cortical variability and asymmetry in normal aging and Alzheimer's disease. Cereb. Cortex 8, 492-509.

Thompson, P.M., Mega, M.S., Woods, P.W., Zoumalan, C.I., Lindshield, C.J., Blanton, R.E., Moussai, J., Holmes, C.J., Cummings, J.L., Toga, A.W., 2001. Cortical change in Alzheimer's disease detected with a disease-specific population-based brain atlas. Cereb. Cortex 11, 1-16.

Yuecel, M., Stuart, G.W., Maruff, P., Velakoulis, D., Crowe, S.F., Savage, G., Pantelis, C., 2001. Hemispheric and gender-related differences in the gross morphology of the anterior cingulate/paracingulate cortex in normal volunteers: an MRI morphometric study. Cereb. Cortex 11, 17-25.

Zucker, S.W., Hummel, R.A., 1981. A 3D edge operator. IEEE T Pattern Anal. 3, 324-331. 